\title{
Adaptación del cuestionario de la personalidad eficaz en estudiantes de secundaria en centros educativos estatales
}

\author{
Adaptation of personality questionnaire effective in high school students in state \\ educational centers
}

Mario Bulnes B. ${ }^{1}$, C. Leni Alvarez T. Carlos Ponce D., Jaime Aliaga T.

Universidad Nacional Mayor de San Marcos. Lima, Perú

(RECIBIDO 30/03/2014, ACEPTADO 29/05/2014)

\begin{abstract}
RESUMEN
El objetivo principal del presente artículo es presentar la adaptación del cuestionario de personalidad eficaz en estudiantes de secundaria de centros educativos estatales de Lima Metropolitana. El constructo teórico del instrumento de personalidad eficaz fue elaborado por Martín del Buey (1997, 2000), en un intento por describir y explicar los factores personales que inciden en el desempeño de los escolares en el nivel educativo secundario. El instrumento original fue elaborado por Fernández, Morís, Marcone, Trigo y Dapelo. La muestra empleada está constituida por 377 alumnos, con edades entre los 14 y 17 años, de los cuales 173 son del sexo femenino y 204 del sexo masculino, de cuarto y quinto año de secundaria. Los resultados obtenidos demuestran que el cuestionario de personalidad eficaz de Martín del Buey presenta validez de constructo, evaluado a través del análisis factorial exploratorio extrayendo la presencia de cuatro factores o constructos, los cuales presentan consistencia interna estimada por los índices de confiabilidad.
\end{abstract}

Palabras clave: personalidad eficaz, estudiantes de secundaria, centros educativos estatales

\begin{abstract}
The main objective of this paper is to present the adaptation of effective personality questionnaire in high school students in state educational centers of Lima city. The theoretical construct of effective Personality instrument was developed by Martin del Buey (1997, 2000) in an attempt to describe and explain the personal factors that affect the performance of students in the secondary education level. The original instrument was developed by Fernández, Moris, Marcone, Wheat and Dapelo. The sample used consists of 377 students, aged between 14 and 17 years, of which 173 are female and 204 male, fourth and fifth grade. The results show that Martin del Buey's effective personality questionnaire has construct validity, assessed through exploratory factor analysis extracting the presence of four factors or constructs, which have internal consistency estimates for the reliability indices.
\end{abstract}

Keywords: effective personality; secondary school students; state education center. 


\section{INTRODUCCIÓN}

Los cambios científicos, tecnológicos y sociales que se vienen dando en los últimos años exigen que la formación recibida por los niños y adolescentes también se reoriente de tal forma que los aprendizajes recibidos en los colegios se adecúen a las nuevas demandas de su contexto inmediato.

En este sentido, habría que recordar que la finalidad educativa está centrada en la formación integral del educando, la cual se puede concretizar en la adquisición de capacidades y actitudes que le permita alcanzar una adecuada adaptación y ajuste personal, escolar y social.

Una forma de responder a estas demandas, tanto sociales como educativas, la constituyen los programas de intervención para niños y adolescentes en contextos escolares. Estos programas permiten conocer a profundidad la problemática educativa a nivel personal, es decir, conocer no solo la manifestación del problema (como el pandillaje, bullying, drogadicción, etc.), sino las condiciones personales que hacen que ciertos alumnos sean vulnerables a determinados factores socioambientales negativos.

En este sentido, Martín del Buey (1997) aplica un programa de entrenamiento en escolares, de manera circular y progresiva, en el que se puede identificar algunas características personales que favorecen o no al ajuste personal. A partir de estas experiencias se plantea la importancia de ciertos aspectos que componen el concepto de personalidad eficaz, constructo teórico que desarrolla el autor mencionado, el cual permite conocer los factores personales que facilitan los éxitos escolares y personales de los adolescentes.

Es así como la necesidad de contar con un instrumento de medida fiable y válido se convirtió en un objetivo imprescindible, de tal forma que permita describir las características personales de los alumnos antes y después de haber participado del programa y, con ello, conocer la efectividad del programa.

Los aspectos que conforman el concepto de personalidad eficaz, a criterio de Martín del Buey, lo constituyen cuatro esferas del yo, los que a continuación se detallan:

Esfera de fortalezas del yo: Autoconcepto

Autoestima

Esfera de demandas del yo: Motivaciones

Expectativas

Atribuciones

Esfera de retos del yo: $\quad$ Afrontamiento de problemas

Toma de decisiones

Esfera de relaciones del yo: Comunicación

Empatía

Asertividad 
El concepto de personalidad eficaz, planteado por Martín del Buey, parte del concepto de autoeficacia desarrollado por Bandura en 1987, quien la define como los juicios que hace cada individuo sobre sus capacidades para alcanzar determinados niveles de rendimiento. En este caso, el concepto se orienta al contexto escolar, por lo que debe asumirse como los juicios que cada alumno realiza sobre sus capacidades con la finalidad de alcanzar determinados niveles de rendimiento académico.

Como señala Pajares (2002; citado por Martín del Buey, Zapico, Moris, Marcone y Dapello, 2004), la autoeficacia afecta al comportamiento humano de la siguiente manera:

Influye en la elección de actividades y conductas.

Determina el grado de esfuerzo a invertir en una actividad, así como su perseverancia, frente a los obstáculos que puedan presentársele.

Influye en los patrones de pensamiento y las reacciones emocionales.

Permite al sujeto ser productor de su propio futuro y no un simple predictor.

En el caso específico de la personalidad eficaz, las características que la definen en términos de rasgos son las siguientes: ansiedad adaptativa, autoestima, autocontrol, perseverancia, introversión estable, extroversión estable. Sin embargo, además de los rasgos señalados, los autores intentaron incorporar otras características ayudándose de referencias bibliográficas, entre ellas se encuentran las siguientes: Asertividad, autoestima, autoconcepto, capacidad de trabajo, confianza en sí mismo, estabilidad emocional, estilos de pensamiento, extraversión, flexibilidad, independencia, iniciativa, locus de control, motivación de logro, optimismo, perseverancia, relaciones sociales, tolerancia a la incertidumbre, toma de riesgos, valores personales, expectativas de autoeficacia, estilo atribucional y estilo de afrontamiento. El cuestionario sobre personalidad eficaz desarrollado por Martín del Buey es justamente un intento de integrar estos aspectos señalados.

En un primer momento, Martín del Buey (2000) construye el cuestionario de personalidad eficaz en contextos educativos con 44 ítems, nutriéndose de las siguientes fuentes:

Autoconcepto Forma 5, de García y Musitu (1999). TEA. Madrid.

Escala de afrontamiento para adolescentes, de Frydenberg y Lewis (1997). TEA. Madrid.

Escala de habilidades sociales, de Gismero González (2000). TEA. Madrid.

Batería Matex (para sujetos de 11 a 15 años). Está formada por los siguientes instrumentos: El cuestionario MAPE-I (motivación hacia el aprendizaje y la ejecución), de Alonso Tapia y Sánchez Ferrer (1992); el cuestionario EAT (estilos atributivos de Alonso Tapia y Sánchez García (1992)) y el cuestionario ECO (expectativas de control), de Alonso Tapia y Arce Saez (1992). 
Batería MAT (para sujetos de 15 a 18 años). Está formada por los siguientes instrumentos: el cuestionario MAPE-II (motivación hacia el aprendizaje y la ejecución), de Montero García y Tapia (1992), y EMA II (escala multidimensional de atribuciones), de Alonso Tapia, Montero García y Mateos Sanz (1992).

En la realización de la adaptación del cuestionario para la realidad de Lima Metropolitana, se han seguido los procesos metodológicos tal como lo realizaran Martín del Buey, Zapico, Moris, Marcone y Dapello (2004) en la construcción del cuestionario de personalidad eficaz en su versión original, quienes lo formularon para estudiantes del nivel secundario. El cuestionario original está compuesto por 58 ítems.

\section{MÉTODO}

\section{Participantes}

La muestra del presente estudio está conformada por 377 alumnos de cuarto y quinto año de secundaria de instituciones educativas estatales de Lima Metropolitana. Como se puede observar en la tabla 1, 193 (51.19\%) alumnos pertenecen al cuarto año de secundaria y $184(48.81 \%)$ pertenecen al quinto año. De ellos, $173(45.89 \%)$ son varones y $204(54.11 \%)$ son mujeres.

Tabla 1. Distribución de frecuencias de la muestra según edad, año de estudio y sexo

\begin{tabular}{cccccc}
\hline \multirow{2}{*}{ Edad } & \multicolumn{2}{c}{ Cuarto año } & \multicolumn{2}{c}{ Quinto año } & \multirow{2}{*}{ Total } \\
\cline { 2 - 5 } & Varón & Mujer & Varón & Mujer & \\
\hline 14 & 7 & 6 & - & - & $13(3.45 \%)$ \\
15 & 75 & 66 & 14 & 19 & $174(46.15 \%)$ \\
16 & 14 & 16 & 36 & 59 & $125(33.16 \%)$ \\
17 & 3 & 6 & 24 & 32 & $65(17.24 \%)$ \\
\hline \multirow{2}{*}{ Total } & 99 & 94 & 74 & 110 & 377 \\
& $(26.26 \%)$ & $(24.93 \%)$ & $(19.63 \%)$ & $(29.18 \%)$ & $(100 \%)$ \\
\hline
\end{tabular}

\section{Descripción del instrumento}

El cuestionario de personalidad eficaz en contextos escolares es la primera versión del instrumento que fuera elaborado por Martín del Buey y que luego se sometió a revisión por el mismo equipo de investigadores, disminuyéndose considerablemente el número de ítems; sin embargo, tomando en consideración las diferencias sociales y culturales de los alumnos españoles y peruanos, es que se ha considerado pertinente en la presente revisión a nuestro contexto educativo, utilizar la versión original del cuestionario en mención. 
Como ya se dijo, el cuestionario consta de 58 ítems, cuyo formato de respuesta es de tipo Lickert y está graduado de uno a cuatro, considerándose las categorías de "estoy totalmente en desacuerdo", "estoy casi en desacuerdo", "estoy casi de acuerdo" y "estoy totalmente de acuerdo", respectivamente.

En el caso de la graduación de las alternativas del cuestionario original, estas iban de cero a cinco, lo que quiere decir que contaba con seis alternativas. En este caso se ha preferido disminuir las alternativas de respuesta de seis a cuatro, a fin de obtener una respuesta más directa y clara sobre lo que el alumno piensa.

Los factores con los que cuenta el instrumento original son cuatro: autorrealización académica, autorrealización socioafectiva, eficacia resolutiva y control académico social. Estos factores también fueron analizados en el presente estudio.

\section{RESULTADOS}

\section{Análisis de la validez del cuestionario general}

En primer lugar se realizó la validez de contenido, mediante el criterio de jueces, para lo cual se contó con la colaboración de 10 expertos tanto en temas de investigación como de personalidad y de investigación educativa. Los resultados evidenciaron valores $\mathrm{V}$ de Aiken superiores a .80 para cada uno de los ítems, con lo que se demostró que el instrumento tiene un alto nivel de validez de contenido.

Seguidamente se procedió a realizar el análisis de validez de constructo mediante el análisis factorial exploratorio, mediante el método de los componentes principales y el método de rotación Oblimin, para lo cual se utilizó como requisito la utilización de adecuación de la muestra de Kaiser-Meyer-Olkin (KMO) y la prueba de esfericidad de Bartlett.

Tabla 2. Valores del determinante, KMO y la prueba de esfericidad de Bartlett

\begin{tabular}{lcc}
\hline Determinante de la matriz & .424 \\
Medidad de adecuación muestral de Kaiser-Meyer-Olkin. & .687 \\
Prueba de esfericidad de Bartlett & Chi -cuadrado & 7817.818 \\
& Df & 320.931 \\
\hline
\end{tabular}

Como se puede observar en la tabla 2, la medida de adecuación del muestreo de Kaiser-Meyer-Olkin (KMO) es de .687 (mayor a .05), el cual puede considerarse como adecuado y la prueba de esfericidad de Bartlett presenta un valor de 320.931, con un nivel de significancia de $.000(\mathrm{p}<.001)$ presentando la intercorrelación requerida para realizar el análisis factorial correspondiente. Estos hallazgos permitieron corroborar la pertinencia de utilizar el análisis factorial en los datos del estudio. 
Luego se procedió a realizar el análisis del gráfico de sedimentación (gráfico 1), con el fin de determinar el número de factores que conformarían la estructura factorial, la cual se señala con una línea diagonal el cambio de pendiente entre los componentes 3 y 5 .

Para llegar a la solución factorial, se utilizó -como método de extracción- el análisis de componentes principales y como método de rotación se utilizó la Normalización Oblimin de Kaiser.

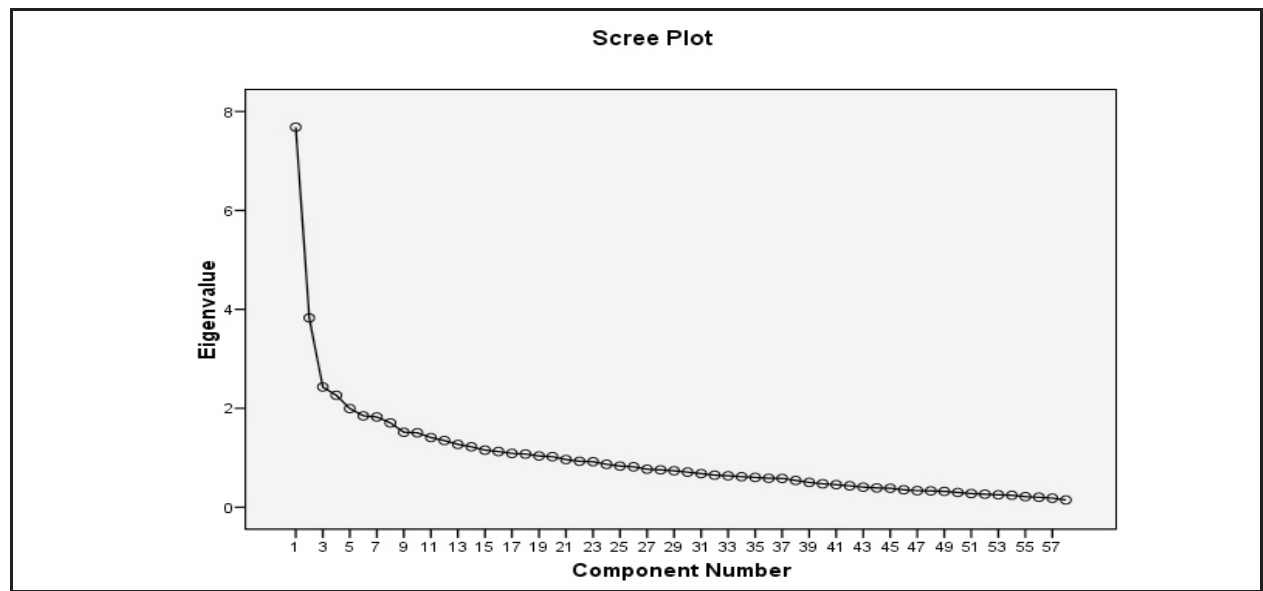

Figura 1. Gráfico de sedimentación del cuestionario de personalidad eficaz

A base del análisis del contenido de los ítems obtenidos de la extracción del análisis factorial, se ha preferido mantener los nombres de los factores que le asignara Martín del Buey al instrumento original. Los resultados de este análisis demostraron ser favorables a la estructura de cuatro factores. El factor 1 explica el $24.865 \%$ de la varianza total, constituido por todos los ítems que corresponden al factor eficacia resolutiva; el factor 2 explica el $20.247 \%$ de la varianza de las puntuaciones del factor control académico social, el factor 3 explica el $14.857 \%$ de la varianza de las puntuaciones del factor autorrealización socioafectiva y el factor 4 explica el $10.031 \%$ de la varianza de las puntuaciones del factor autorrealización académica (tabla 3 ).

Tabla 3. Datos descriptivos de los factores de la personalidad eficaz

\begin{tabular}{lccc}
\hline \multicolumn{1}{c}{ Factores } & $\mathrm{M}$ & D.E. & Varianza explicada \\
\hline $\begin{array}{l}\text { 1. Eficacia resolutiva } \\
\text { 2. Control académico social }\end{array}$ & 55.59 & 6.518 & 24.865 \\
$\begin{array}{l}\text { 3. Autorrealización } \\
\text { socioafectiva }\end{array}$ & 24.23 & 5.802 & 20.247 \\
$\begin{array}{l}\text { 4. Autorrealización } \\
\text { académica }\end{array}$ & 46.92 & 5.839 & 14.857 \\
\hline
\end{tabular}


A continuación se presentan los resultados obtenidos en el análisis factorial exploratorio en función de la distribución de los ítems por factores y al peso que tiene cada ítem dentro del factor (tablas 4, 5, 6 y 7).

Tabla 4. Ítems que componen el factor eficacia resolutiva

\begin{tabular}{|c|c|c|}
\hline $\mathrm{N}^{\circ}$ & Ítems & Peso por ítem \\
\hline 3 & Creo que tendré problemas en mis relaciones con los demás. & ,463 \\
\hline 4 & Me siento incapaz de resolver mis problemas. &, 535 \\
\hline 9 & Mis éxitos en los estudios se deben a la suerte. &, 512 \\
\hline 18 & Mis fracasos en las relaciones con los demás se deben a que no les gusto. &, 529 \\
\hline 24 & Estudio porque con ello puedo evitarme muchos problemas. & ,470 \\
\hline 25 & Mis éxitos en los estudios se deben a que los cursos son fáciles. & ,462 \\
\hline 27 & Mis fracasos en las relaciones con los demás se deben a mi falta de habilidad. & ,497 \\
\hline 32 & Cuando tengo un problema evito buscar ayuda. & ,322 \\
\hline 33 & Estudio para no sentirme inferior a mis compañeros. & ,489 \\
\hline 34 & Mis fracasos en las relaciones con los demás se deben a mi falta de esfuerzo. &, 513 \\
\hline 37 & Cuando tengo un problema no hago nada para resolverlo. &, 501 \\
\hline 43 & Cuando tengo que tomar una decisión me pongo tan nervioso que al final no hago nada. &, 554 \\
\hline 45 & Mis éxitos en las relaciones con los demás se deben a que son ellos los que toman la iniciativa. & ,422 \\
\hline 46 & Los problemas que tengo son por mi culpa. &, 313 \\
\hline 48 & Mis fracasos en los estudios se deben a la mala suerte. &, 501 \\
\hline 52 & Estudio para obtener premios de mis padres. &, 546 \\
\hline 54 & Cuando tengo que tomar una decisión elijo la primera opción que se me ocurre. & ,439 \\
\hline 55 & Mis fracasos en los estudios se deben a mis profesores. & ,431 \\
\hline
\end{tabular}

Tabla 5. Ítems que componen el factor control académico social

\begin{tabular}{llr}
\hline $\mathrm{N}^{\circ}$ & \multicolumn{1}{c}{ Items } & Peso por ítem \\
\hline 20 & Cuando tengo un problema suelo pedir ayuda. &, 321 \\
22 & Hago amigos con facilidad. &, 616 \\
36 & Expreso mis opiniones abiertamente. &, 397 \\
41 & Estoy a gusto con mi aspecto físico. &, 339 \\
42 & Mis éxitos en las relaciones con los demás se deben a mi habilidad para hacer amigos. &, 630 \\
49 & Me siento querido por mi familia. &, 524 \\
51 & Me da vergüenza hablar en público. &,- 314 \\
53 & Creo que tendré éxito en mis relaciones con los demás. &, 539 \\
56 & Creo que soy una persona valiosa para los otros. &, 408 \\
\hline
\end{tabular}

Tabla 6. Ítems que componen el factor autorrealización socioafectiva

\begin{tabular}{clc}
\hline $\mathrm{N}^{\circ}$ & \multicolumn{1}{c}{ Ítems } & Peso por ítem \\
\hline 11 & Cuando tengo un problema trato de ver el lado positivo de la situación. &, 489 \\
12 & Analizo las posibles consecuencias de mis decisiones antes de tomarlas. &, 559 \\
13 & Me acepto tal y como soy, con mis cualidades y defectos. &, 441 \\
16 & Para tomar decisiones reúno toda la información que puedo encontrar. &, 449 \\
17 & Sé decir NO cuando creo que debo hacerlo. &, 369
\end{tabular}




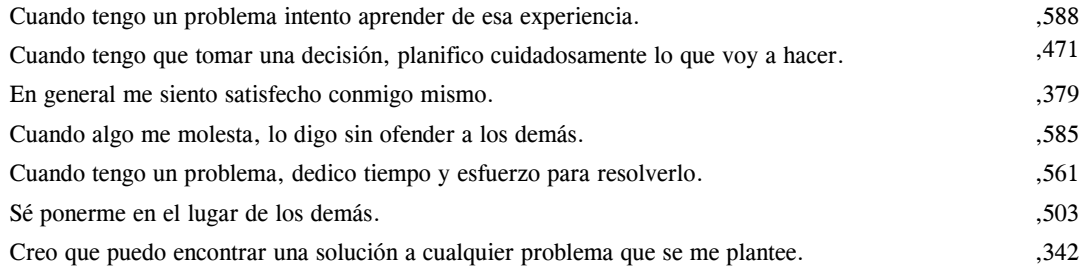

Tabla 7. Ítems que componen el factor autorrealización académica

\begin{tabular}{clc}
\hline $\mathrm{N}^{\circ}$ & \multicolumn{1}{c}{ Items } & Peso por ítem \\
\hline 1 & Me considero un buen estudiante. &, 598 \\
8 & Estudio porque me interesa aprender cosas nuevas. &, 530 \\
10 & Creo que aprobaré todos los cursos de este año. &, 409 \\
14 & Mis fracasos en los estudios se deben a mi falta de esfuerzo. &,- 322 \\
15 & Creo que tendré éxito en mis estudios futuros. &, 521 \\
19 & Nadie me obliga a estudiar, estudio porque quiero hacerlo. &, 350 \\
23 & Estudio porque me gusta superar retos. &, 548 \\
30 & Mis éxitos en los estudios se deben a mi capacidad. &, 571 \\
38 & Mis éxitos en los estudios se deben a mi esfuerzo. &, 558 \\
44 & Estudio para sacar buenas notas. &, 453 \\
57 & Estudio porque me gusta comprobar que soy capaz de hacerlo. &, 458 \\
\hline
\end{tabular}

En el análisis factorial, se excluyeron todos aquellos ítems con valores menores a .30 , quedando excluidos los ítems $2,5,6,7,21,29,35,50$, en la medida que no presentaron un valor discriminatorio entre los sujetos, por lo tanto, no contribuían a la coherencia interna de la prueba.

\section{Análisis de la confiabilidad de la prueba}

El análisis de la prueba completa se realizó mediante la prueba de correlación ítemtest corregida (RITC) y mediante el coeficiente de confiabilidad por consistencia interna expresado por el coeficiente alfa de Cronbach.

Los valores ritc encontrados oscilan entre .212 y .525 , es decir, son superiores a .20 (Kline, 1993) en el análisis de cada factor (tabla 8). En el caso del ítem 14, este fue eliminado ya que alcanzó un valor por debajo de lo esperado.

Tabla 8. Coeficientes de relación ítem-test corregido según factores

\begin{tabular}{ccccccc}
\hline Factor & Ítem & RITC & Alfa si se elimina ítem & Ítem & RITC & Alfa si se elimina ítem \\
\hline & 3 &, 434 &, 800 & 34 &, 459 &, 798 \\
& 4 & 412 &, 801 & 37 &, 438 &, 800 \\
Eficacia & 9 &, 404 &, 802 & 43 &, 469 &, 797 \\
resolutiva & 18 &, 499 &, 797 & 45 &, 356 &, 804 \\
& 24 &, 309 &, 808 & 46 &, 254 &, 811 \\
& 25 &, 351 &, 804 & 48 &, 463 &, 799 \\
& 32 &, 483 &, 796 & 52 &, 281 &, 800 \\
& 33 &, 401 &, 809 & 54 &, 344 &, 805 \\
\hline
\end{tabular}


Mario Bulnes B.1, C. Leni Alvarez T. Carlos Ponce D., Jaime Aliaga T.

\begin{tabular}{|c|c|c|c|c|c|c|}
\hline & 11 & ,366 & ,745 & 28 &, 379 & ,743 \\
\hline 2 & 12 & ,477 & ,732 & 31 &, 370 & ,744 \\
\hline \multirow{4}{*}{ Control } & 13 & ,355 & ,746 & 39 & ,494 & ,729 \\
\hline & 16 & ,422 & ,738 & 40 & ,469 & ,733 \\
\hline & 17 & ,295 & ,754 & 47 &, 374 & ,745 \\
\hline & 26 &, 457 & ,735 & 58 &, 268 & ,755 \\
\hline \multirow[b]{2}{*}{3} & 20 &, 212 & ,677 & 49 &, 354 & ,646 \\
\hline & 22 &, 454 & ,624 & 51 &, 235 & ,682 \\
\hline \multirow{3}{*}{$\begin{array}{c}\text { Autorrealización } \\
\text { socioafectiva }\end{array}$} & 36 & ,366 & ,644 & 53 & ,498 & ,617 \\
\hline & 41 & ,261 & ,664 & 56 & ,401 & ,637 \\
\hline & 42 &, 437 & ,628 & & & \\
\hline \multirow[b]{2}{*}{4} & 1 & 450 & ,706 & 23 & ,385 & ,712 \\
\hline & 8 & ,403 & ,710 & 30 &, 502 & 695 \\
\hline \multirow{3}{*}{$\begin{array}{c}\text { Autorrealización } \\
\text { académica }\end{array}$} & 10 & ,381 & ,714 & 38 &, 525 & 692 \\
\hline & 15 & ,437 & ,705 & 44 &, 316 & ,725 \\
\hline & 19 & ,267 &, 735 & 57 &, 348 & ,718 \\
\hline
\end{tabular}

En cuanto a los valores alfa de Cronbach, el factor eficacia resolutiva alcanza un valor de .811 con 18 ítems, el factor control académico social alcanza una valor de .758 con 12 ítems, el factor autorrealización socioefectiva alcanza un valor de .673 con 9 ítems y el factor autorrealización académica alcanza un valor de .732 con 10 ítems (tabla 9). Con estos datos se demuestra que el cuestionario de personalidad eficaz es una prueba altamente confiable.

Tabla 9. Coeficientes alfa de Cronbach de los factores y escala total

\begin{tabular}{ccc}
\hline Factor & Dimensiones & Alpha de Cronbach \\
\hline 1 & Eficacia resolutiva (18 ítems) &, 811 \\
2 & Control académico social (12 ítems) &, 758 \\
3 & Autorrealización socioafectiva (9 ítems) &, 673 \\
4 & Autorrealización académica (10 ítems) &, 732 \\
\hline
\end{tabular}

Finalmente, los factores del cuestionario de personalidad eficaz quedaron constituidos por los siguientes ítems:

\begin{tabular}{ccl}
\hline Factor & $N^{\circ}$ de ítem & \multicolumn{1}{c}{ Ítem } \\
\hline & 2 & Creo que tendré problemas en mis relaciones con los demás. \\
& 3 & Me siento incapaz de resolver mis problemas. \\
& 5 & Mis éxitos en los estudios se deben a la suerte. \\
& 13 & Mis fracasos en las relaciones con los demás se deben a que no les gusto. \\
& 19 & Estudio porque con ello puedo evitarme muchos problemas. \\
& 21 & Mis éxitos en los estudios se deben a que los cursos son fáciles. \\
& 25 & Cuando tengo un problema evito buscar ayuda. \\
Eficacia & 26 & Estudio para no sentirme inferior a mis compañeros. \\
resolutiva & 27 & Mis fracasos en las relaciones con los demás se deben a mi falta de esfuerzo. \\
& 29 & Cuando tengo un problema no hago nada para resolverlo. \\
& 35 & Cuando tengo que tomar una decisión me pongo tan nervioso que al final no hago nada. \\
& 37 & Mis éxitos en las relaciones con los demás se deben a que son ellos los que toman la iniciativa. \\
& 38 & Los problemas que tengo son por mi culpa. \\
& 40 & Mis fracasos en los estudios se deben a la mala suerte. \\
& 43 & Estudio para obtener premios por parte de mis padres. \\
& 45 & Cuando tengo que tomar una decisión, elijo la primera opción que se me ocurre. \\
& 46 & Mis fracasos en los estudios se deben a mis profesores.
\end{tabular}




\begin{tabular}{|c|c|c|}
\hline \multirow{12}{*}{$\begin{array}{c}2 \\
\text { Control } \\
\text { académico social }\end{array}$} & 7 & Cuando tengo un problema, trato de ver el lado positivo de la situación. \\
\hline & 8 & Analizo las posibles consecuencias de mis decisiones antes de tomarlas. \\
\hline & 9 & Me acepto tal y como soy, con mis cualidades y defectos. \\
\hline & 11 & Para tomar decisiones reúno toda la información que puedo encontrar. \\
\hline & 12 & Sé decir NO cuando creo que debo hacerlo. \\
\hline & 20 & Cuando tengo un problema, intento aprender de esa experiencia. \\
\hline & 22 & Cuando tengo que tomar una decisión, planifico cuidadosamente lo que voy a hacer. \\
\hline & 24 & En general, me siento satisfecho conmigo mismo. \\
\hline & 31 & Cuando algo me molesta, lo digo sin ofender a los demás. \\
\hline & 32 & Cuando tengo un problema, dedico tiempo y esfuerzo para resolverlo. \\
\hline & 39 & Sé ponerme en el lugar de los demás. \\
\hline & 49 & Creo que puedo encontrar una solución a cualquier problema que se me plantee. \\
\hline \multirow{9}{*}{$\begin{array}{l}3 \\
\text { Autorrealización } \\
\text { socioafectiva }\end{array}$} & 15 & Cuando tengo un problema suelo pedir ayuda. \\
\hline & 16 & Hago amigos con facilidad. \\
\hline & 28 & Expreso mis opiniones abiertamente. \\
\hline & 33 & Estoy a gusto con mi aspecto físico. \\
\hline & 34 & Mis éxitos en las relaciones con los demás se deben a mi habilidad para hacer amigos. \\
\hline & 41 & Me siento querido por mi familia. \\
\hline & 42 & Me da vergüenza hablar en público. \\
\hline & 44 & Creo que tendré éxito en mis relaciones con los demás. \\
\hline & 47 & Creo que soy una persona valiosa para los otros. \\
\hline \multirow{10}{*}{$\begin{array}{c}4 \\
\text { Autorrealización } \\
\text { académica }\end{array}$} & 1 & Me considero un buen estudiante. \\
\hline & 4 & Estudio porque me interesa aprender cosas nuevas. \\
\hline & 6 & Creo que aprobaré todos los cursos de este año. \\
\hline & 10 & Creo que tendré éxito en mis estudios futuros. \\
\hline & 14 & Nadie me obliga a estudiar, estudio porque quiero hacerlo. \\
\hline & 17 & Estudio porque me gusta superar retos. \\
\hline & 23 & Mis éxitos en los estudios se deben a mi capacidad. \\
\hline & 30 & Mis éxitos en los estudios se deben a mi esfuerzo. \\
\hline & 36 & Estudio para sacar buenas notas. \\
\hline & 48 & Estudio porque me gusta comprobar que soy capaz de hacerlo. \\
\hline
\end{tabular}

\section{Baremos del cuestionario de personalidad eficaz}

Los baremos han sido elaborados a base de rangos percentiles, los cuales son los puntajes expresados del grupo normativo que, en este caso, corresponden a alumnos de cuarto y quinto año de secundaria de instituciones educativas estatales de Lima Metropolitana. Esta norma se aplicó dividiendo las puntuaciones directas en cien unidades que conforman la escala de rango ordinal. Los baremos son presentados de acuerdo a la muestra total, seguidamente se presentan los baremos según grado escolar y según sexo.

Tabla 10. Rangos percentilares del cuestionario de personalidad eficaz en escolares de cuarto y quinto año de educación secundaria - Muestra total

\begin{tabular}{ccccccc}
\hline Percentil & Factor 1 & Factor 2 & Factor 3 & Factor 4 & Personalidad eficaz & Percentil \\
\hline 1 & $18-20$ & $12-24$ & $9-18$ & $10-20$ & $49-103$ & 1 \\
2 & 21 & $25-27$ & $19-20$ & $21-23$ & $104-113$ & 2 \\
3 & 22 & $28-29$ & & 24 & $114-115$ & 3 \\
4 & 23 & 30 & 21 & 25 & 116 & 4 \\
5 & 24 & & & & $117-120$ & 5
\end{tabular}


Mario Bulnes B.1, C. Leni Alvarez T. Carlos Ponce D., Jaime Aliaga T.

\begin{tabular}{|c|c|c|c|c|c|c|}
\hline 9 & 25 & $31-33$ & 22 & 26 & $121-122$ & 9 \\
\hline 12 & & 34 & 23 & 27 & $123-124$ & 12 \\
\hline 15 & 26 & & 24 & 28 & $125-126$ & 15 \\
\hline 17 & & 35 & 25 & & 127 & 17 \\
\hline 21 & $27-28$ & & & 29 & 128 & 21 \\
\hline 23 & & 36 & & 30 & 129 & 23 \\
\hline 26 & & & & & 130 & 26 \\
\hline 30 & 29 & 37 & 26 & 31 & 131 & 30 \\
\hline 32 & 30 & & & & 132 & 32 \\
\hline 38 & 31 & 38 & 27 & 32 & 133 & 38 \\
\hline 41 & 32 & & & & 134 & 41 \\
\hline 43 & & & & 33 & 135 & 43 \\
\hline 49 & $33-34$ & 39 & 28 & & 136 & 49 \\
\hline 50 & 35 & 40 & 29 & 34 & 137 & 50 \\
\hline 54 & 36 & 41 & & 35 & 138 & 54 \\
\hline 58 & 37 & & & & 139 & 58 \\
\hline 62 & 38 & 42 & 30 & 36 & 140 & 62 \\
\hline 66 & 39 & & & & $141-142$ & 66 \\
\hline 72 & 40 & 43 & 31 & 37 & 143 & 72 \\
\hline 75 & 41 & & & & 144 & 75 \\
\hline 78 & 42 & 44 & & & 145 & 78 \\
\hline 82 & 43 & & 32 & 38 & 146 & 82 \\
\hline 85 & 44 & 45 & & & $147-148$ & 85 \\
\hline 88 & $45-47$ & 46 & & & 149 & 88 \\
\hline 91 & 48 & & 33 & 39 & $150-151$ & 91 \\
\hline 93 & 49 & 47 & & & 152 & 93 \\
\hline 95 & $50-51$ & & & & $153-154$ & 95 \\
\hline 96 & $52-53$ & & 34 & 40 & $155-157$ & 96 \\
\hline 98 & $54-55$ & & 35 & & $158-165$ & 98 \\
\hline 99 & $56-72$ & 48 & 36 & & $166-196$ & 99 \\
\hline Media & 35.22 & 39.56 & 28,28 & 33.48 & 136.54 & Media \\
\hline Mediana & 35.00 & 40.00 & 29.00 & 34.00 & 137.00 & Mediana \\
\hline Moda & 29 & 41 & 29 & 34 & 133 & Moda \\
\hline Desv. típ. & 8.123 & 4.922 & 3.563 & 4.373 & 11.011 & Desv. típ \\
\hline Sujetos & 377 & 377 & 377 & 377 & 377 & Sujetos \\
\hline
\end{tabular}

Tabla 11. Rangos percentilares del cuestionario de personalidad eficaz en escolares de cuarto año de educación secundaria

\begin{tabular}{ccccccc}
\hline Percentil & Factor 1 & Factor 2 & Factor 3 & Factor 4 & Personalidad eficaz & Percentil \\
\hline 1 & $18-19$ & $12-23$ & $9-18$ & $10-20$ & $49-105$ & 1 \\
2 & 20 & $24-27$ & 19 & 21 & $106-110$ & 2 \\
3 & 21 & $28-29$ & & $22-23$ & $111-114$ & 3 \\
4 & 22 & & & 24 & 115 & 4 \\
5 & 24 & $20-21$ & & 25 & $116-119$ & 5 \\
\hline 8 & 25 & $30-31$ & 22 & 26 & 122 & 8 \\
9 & 26 & $33-34$ & 23 & 27 & 123 & 9 \\
12 & & & & & & 12
\end{tabular}


AdAPTACIÓN DEL CUESTIONARIO DE LA PERSONALIDAD EFICAZ EN ESTUDIANTES DE SECUNDARIA EN CENTROS EDUCATIVOS ESTATALES

\begin{tabular}{|c|c|c|c|c|c|c|}
\hline 15 & & & $24-25$ & 28 & $124-125$ & 15 \\
\hline 18 & 27 & 35 & & & $126-127$ & 18 \\
\hline 22 & 28 & & & 29 & 128 & 22 \\
\hline 26 & & & 26 & 30 & 129 & 26 \\
\hline 30 & 29 & 36 & & & 130 & 30 \\
\hline 33 & 30 & 37 & & & 131 & 33 \\
\hline 38 & 31 & 38 & 27 & 31 & 132 & 38 \\
\hline 42 & 32 & & & 32 & 133 & 42 \\
\hline 45 & & & & 33 & 134 & 45 \\
\hline 48 & 33 & 39 & 28 & & $135-136$ & 48 \\
\hline 50 & 34 & 40 & 29 & 34 & 137 & 50 \\
\hline 54 & 35 & 41 & & & 138 & 54 \\
\hline 55 & 36 & & & 35 & & 55 \\
\hline 60 & 37 & & 30 & & 139 & 60 \\
\hline 63 & 38 & & & 36 & 140 & 63 \\
\hline 65 & & 42 & & & 141 & 65 \\
\hline 68 & 39 & & 31 & & 142 & 68 \\
\hline 72 & 40 & 43 & & 37 & 143 & 72 \\
\hline 77 & 41 & 44 & & & $144-145$ & 77 \\
\hline 81 & $42-43$ & & 32 & 38 & 146 & 81 \\
\hline 86 & 44 & 45 & & & 147 & 86 \\
\hline 88 & 45 & & 33 & 39 & 148 & 88 \\
\hline 90 & $46-47$ & 46 & & & $149-150$ & 90 \\
\hline 93 & 48 & & & & 151 & 93 \\
\hline 95 & 49 & 47 & & & $152-153$ & 95 \\
\hline 96 & $50-53$ & & 34 & 40 & $154-155$ & 96 \\
\hline 98 & 54 & & & & $156-158$ & 98 \\
\hline 99 & $55-72$ & 48 & $35-36$ & & $159-196$ & 99 \\
\hline Media & 34.86 & 39.38 & 28.39 & 33.24 & 135.87 & Media \\
\hline Mediana & 34.00 & 40.00 & 29.00 & 34.00 & 137.00 & Mediana \\
\hline Moda & 27 & 41 & 29 & 34 & 138 & Moda \\
\hline Desv. típ. & 7.731 & 4.929 & 3.562 & 4.571 & 10.549 & Desv. típ \\
\hline Sujetos & 193 & 193 & 193 & 193 & 193 & Sujetos \\
\hline
\end{tabular}

Tabla 12. Rangos percentilares del cuestionario de personalidad eficaz en escolares de quinto año de educación secundaria

\begin{tabular}{|c|c|c|c|c|c|c|}
\hline Percentil & Factor 1 & Factor 2 & Factor 3 & Factor 4 & Personalidad eficaz & Percentil \\
\hline 1 & $18-20$ & $12-23$ & $9-16$ & $10-18$ & $49-98$ & 1 \\
\hline 2 & 21 & $24-28$ & $17-19$ & & $99-113$ & 2 \\
\hline 3 & & 29 & & & $114-115$ & 3 \\
\hline 4 & $22-23$ & 30 & $20-21$ & $19-26$ & $116-117$ & 4 \\
\hline 5 & & 31 & 22 & & $118-119$ & 5 \\
\hline 6 & 24 & 32 & & & $120-121$ & 6 \\
\hline 9 & & 33 & & 27 & 122 & 9 \\
\hline 10 & 25 & & 23 & & 123 & 10 \\
\hline 12 & & 34 & & 28 & 124 & 12 \\
\hline 14 & & & 24 & & 125 & 14 \\
\hline
\end{tabular}


Mario Bulnes B.1, C. Leni Alvarez T. Carlos Ponce D., Jaime Aliaga T.

\begin{tabular}{|c|c|c|c|c|c|c|}
\hline 16 & 26 & 35 & & 29 & 126 & 16 \\
\hline 17 & & & & & 127 & 17 \\
\hline 19 & 27 & 36 & 25 & & $128-129$ & 19 \\
\hline 25 & 28 & & & 30 & $130-131$ & 25 \\
\hline 31 & $29-31$ & 37 & 26 & 31 & 132 & 31 \\
\hline 35 & & 38 & 27 & 32 & 133 & 35 \\
\hline 40 & 32 & & & 33 & $134-135$ & 40 \\
\hline 47 & $33-34$ & 39 & 28 & & 136 & 47 \\
\hline 50 & 35 & 40 & 29 & 34 & 137 & 50 \\
\hline 52 & 36 & & & 35 & 138 & 52 \\
\hline 55 & & 41 & & & 139 & 55 \\
\hline 60 & 37 & 42 & 30 & 36 & 140 & 60 \\
\hline 65 & 38 & & & & 141 & 65 \\
\hline 68 & 39 & & & & $142-143$ & 68 \\
\hline 71 & 40 & 43 & & 37 & 144 & 71 \\
\hline 76 & $41-42$ & & 31 & & 145 & 76 \\
\hline 79 & 43 & 44 & & 38 & $146-147$ & 79 \\
\hline 85 & $44-45$ & 45 & 32 & & $148-149$ & 85 \\
\hline 88 & $46-47$ & & & & 150 & 88 \\
\hline 90 & 48 & 46 & & & 151 & 90 \\
\hline 92 & 49 & & & 39 & 152 & 92 \\
\hline 93 & $50-52$ & 47 & 33 & & $153-155$ & 93 \\
\hline 95 & $53-54$ & & & & $156-157$ & 95 \\
\hline 97 & 55 & & 34 & & $158-165$ & 97 \\
\hline 98 & $56-60$ & & 35 & 40 & $166-169$ & 98 \\
\hline 99 & $61-72$ & 48 & 36 & & $170-196$ & 99 \\
\hline Media & 35.59 & 39.74 & 28.17 & 33.73 & 137.24 & Media \\
\hline Mediana & 35.00 & 40.00 & 29.00 & 34.00 & 137.00 & Mediana \\
\hline Moda & 29 & 39 & 29 & 34 & 133 & Moda \\
\hline Desv. típ. & 8.520 & 4.921 & 3.572 & 4.153 & 11.462 & Desv. típ. \\
\hline Sujetos & 184 & 184 & 184 & 184 & 184 & Sujetos \\
\hline
\end{tabular}

Tabla 13. Rangos percentilares del cuestionario de personalidad eficaz en escolares de cuarto y quinto año de educación secundaria - Varones

\begin{tabular}{ccccccc}
\hline Percentil & Factor 1 & Factor 2 & Factor 3 & Factor 4 & Personalidad eficaz & Percentil \\
\hline 1 & $18-21$ & $12-22$ & $9-15$ & $10-17$ & $49-97$ & 1 \\
2 & 22 & $23-26$ & $16-18$ & $18-19$ & $98-108$ & 2 \\
3 & & & 19 & $20-21$ & $109-114$ & 3 \\
4 & $23-24$ & $27-29$ & 20 & 22 & $115-119$ & 4 \\
5 & & & & $23-24$ & 120 & 5 \\
\hline 7 & 25 & $30-31$ & $21-22$ & 25 & 121 & 7 \\
12 & $26-27$ & $32-33$ & 23 & 26 & 122 & 12 \\
\hline 14 & 28 & & 24 & 27 & 123 & 14 \\
15 & & 34 & & & $124-125$ & 15 \\
18 & 29 & & 25 & & $126-127$ & 18 \\
\hline 22 & 30 & 35 & & 28 & 128 & 22 \\
25 & 31 & & & 29 & 129 & 25
\end{tabular}


AdAPTACIÓN DEL CUESTIONARIO DE LA PERSONALIDAD EFICAZ EN ESTUDIANTES DE SECUNDARIA EN CENTROS EDUCATIVOS ESTATALES

\begin{tabular}{|c|c|c|c|c|c|c|}
\hline 30 & 32 & 36 & 26 & 30 & $130-131$ & 30 \\
\hline 35 & 33 & 37 & 27 & 31 & 132 & 35 \\
\hline 41 & 34 & 38 & & & 133 & 41 \\
\hline 44 & 35 & & & & $134-135$ & 44 \\
\hline 50 & 36 & 39 & 28 & $32-33$ & 136 & 50 \\
\hline 52 & 37 & 40 & & 34 & 137 & 52 \\
\hline 58 & 38 & 41 & 29 & & 138 & 58 \\
\hline 62 & 39 & & & 35 & 139 & 62 \\
\hline 66 & 40 & & 30 & & 140 & 66 \\
\hline 70 & 41 & 42 & 31 & 36 & $141-142$ & 70 \\
\hline 74 & 42 & & & & 143 & 74 \\
\hline 77 & 43 & 43 & & 37 & $144-145$ & 77 \\
\hline 82 & 44 & & & & $146-147$ & 82 \\
\hline 85 & 45 & 44 & & & 148 & 85 \\
\hline 87 & & & 32 & 38 & 149 & 87 \\
\hline 90 & 47 & 45 & & & 150 & 90 \\
\hline 92 & $48-49$ & 46 & & & & 92 \\
\hline 93 & 50 & & $33-34$ & & $151-152$ & 93 \\
\hline 95 & $51-53$ & & & 39 & $153-154$ & 95 \\
\hline 96 & $54-55$ & $47-48$ & & & 155 & 96 \\
\hline 97 & & & & & $156-161$ & 97 \\
\hline 98 & $56-61$ & & 35 & & $162-170$ & 98 \\
\hline 99 & $62-72$ & & 36 & 40 & $171-196$ & 99 \\
\hline Media & 36.83 & 38.89 & 27.99 & 32.35 & 136.05 & Media \\
\hline Mediana & 36.00 & 39.00 & 28.00 & 33.00 & 136.00 & Mediana \\
\hline Moda & 36 & 39 & 28 & 34 & 133 & Moda \\
\hline Desv. típ. & 7.956 & 4.983 & 3.575 & 4.661 & 11.489 & Desv. típ. \\
\hline Sujetos & 173 & 173 & 173 & 173 & 173 & Sujetos \\
\hline
\end{tabular}

Tabla 14. Rangos percentilares del cuestionario de personalidad eficaz en escolares de cuarto y quinto año de educación secundaria - Mujeres

\begin{tabular}{ccccccc}
\hline Percentil & Factor 1 & Factor 2 & Factor 3 & Factor 4 & Personalidad eficaz & Percentil \\
\hline 1 & $18-19$ & $12-23$ & $9-18$ & $10-24$ & $49-110$ & 1 \\
2 & 20 & $24-27$ & & & $111-113$ & 2 \\
4 & 21 & $28-29$ & $19-21$ & $25-26$ & $114-115$ & 4 \\
5 & 22 & & & & 116 & 5 \\
6 & 23 & $30-31$ & & & $117-120$ & 6 \\
\hline 8 & 24 & $32-33$ & 22 & 27 & 121 & 8 \\
10 & & 34 & & 28 & $122-123$ & 10 \\
11 & 25 & & 23 & & 124 & 11 \\
\hline 15 & & 35 & 24 & 29 & $125-126$ & 15 \\
17 & 26 & & 25 & 30 & 127 & 17 \\
21 & & 36 & & & $128-129$ & 21 \\
23 & 27 & & & 31 & 130 & 23 \\
\hline 28 & 28 & 37 & 26 & & 131 & 28 \\
30 & & & & 32 & 132 & 30 \\
35 & & 38 & 27 & 33 &
\end{tabular}


Mario Bulnes B.1, C. Leni Alvarez T. Carlos Ponce D., Jaime Aliaga T.

\begin{tabular}{|c|c|c|c|c|c|c|}
\hline 39 & $29-30$ & & & & 134 & 39 \\
\hline 42 & & & 28 & & 135 & 42 \\
\hline 47 & 31 & & & 34 & 136 & 47 \\
\hline 50 & $32-33$ & $40-41$ & 29 & $35-36$ & $137-138$ & 50 \\
\hline 55 & 34 & & & & 139 & 55 \\
\hline 58 & 35 & 42 & 30 & & 140 & 58 \\
\hline 63 & 36 & & & 37 & 141 & 63 \\
\hline 68 & 37 & 43 & & & 142 & 68 \\
\hline 70 & 38 & & 31 & & 143 & 70 \\
\hline 74 & 39 & 44 & & & 144 & 74 \\
\hline 75 & 40 & & 32 & 38 & 145 & 75 \\
\hline 80 & $41-42$ & 45 & & & 146 & 80 \\
\hline 85 & 43 & & & 39 & $147-148$ & 85 \\
\hline 88 & 44 & 46 & 33 & & $149-150$ & 88 \\
\hline 90 & $45-46$ & & & & 151 & 90 \\
\hline 92 & $47-48$ & 47 & & & 152 & 92 \\
\hline 93 & 49 & & & & 153 & 93 \\
\hline 95 & $50-51$ & & & 40 & 154 & 95 \\
\hline 96 & 52 & & 34 & & $155-157$ & 96 \\
\hline 98 & $53-55$ & 48 & 35 & & $158-165$ & 98 \\
\hline 99 & $56-72$ & & 36 & & $166-196$ & 99 \\
\hline Media & 33.85 & 40.12 & 28.53 & 34.45 & 136.96 & Media \\
\hline Mediana & 33.00 & 41.00 & 29.00 & 35.00 & 137.00 & Mediana \\
\hline Moda & 29 & 41 & 29 & 37 & 133 & Moda \\
\hline Desv. típ. & 8.032 & 4.810 & 3.543 & 3.870 & 10.599 & Desv. típ. \\
\hline Sujetos & 204 & 204 & 204 & 204 & 204 & Sujetos \\
\hline
\end{tabular}

\section{RESULTADOS}

El presente estudio ha establecido la adaptación del cuestionario de personalidad eficaz en escolares de cuarto y quinto año de secundaria de instituciones educativas estatales de Lima Metropolitana, y para ello se ha contado con una muestra de 377 alumnos, con edades entre los 14 y 17 años, de los cuales 173 son del sexo femenino y 204 del sexo masculino.

Se procedió a realizar el análisis de validez de contenido con base en el criterio de jueces, en el que se obtuvo valores $\mathrm{V}$ de Aiken por encima de .80, seguidamente se estableció la validez de constructo mediante el análisis factorial exploratorio, para lo cual se utilizó como requisito la utilización de adecuación de la muestra de Kaiser-Meyer-Olkin (KMO) y la prueba de esfericidad de Bartlett, obteniendo luego de realizar el análisis del gráfico de sedimentación la conformación de cuatro factores.

Al realizar un nuevo análisis de ítems con el cuestionario adaptado, se observa que en todos los casos los valores de la correlación ítem-test corregida obtienen coeficientes mayores a .20 y un alfa de Cronbach por encima de .70 (Kline, 1993), lo que indica que el cuestionario adaptado que evalúa la personalidad eficaz en escolares es un instrumento confiable. 
Los factores encontrados, que además confirman la investigación anterior realizada por Martín del Buey, son los siguientes: eficacia resolutiva, control académico social, autorrealización académica, autorrealización socioafectiva.

\section{DISCUSIÓN}

El análisis psicométrico de la prueba para su adaptación alcanzó la validez y confiabilidad requerida para ser utilizado como instrumento de medición en otros trabajos de investigación de esta naturaleza en nuestro medio.

El nombre de cada factor son los mismos que asignó Martín del Buey en la elaboración del cuestionario de personalidad eficaz, pero los ítems para la adaptación limeña son en un número de 49 ítems. Se encontró que la estructura factorial de los ítems aplicados podría ser explicado por la presencia de cuatro factores. Al respecto fueron excluidos los ítems $2,5,6,7,14,21,29,35,50$, debido a su bajo poder discriminatorio.

Se presentan los baremos respectivos tomando como referencias el grado estudio y el sexo, así como un baremo sobre la base de la muestra total; utilizando los puntajes convertidos en término de percentiles, lo que facilita la lectura de los puntajes directos.

En términos generales, estos resultados confirman que los subtests de la prueba son válidos y confiables. Por ello, cumplen con el propósito principal del estudio, en la medida que ponen al alcance de psicólogos, educadores y otros profesionales vinculados con el tema, un cuestionario con base teórica y metodológica para hacer aplicada en nuestro medio.

\section{CONCLUSIONES}

1. El cuestionario de personalidad eficaz, de Martín del Buey, ha demostrado tener validez de contenido y de constructo, evaluado a través del criterio de jueces y del análisis factorial exploratorio, que de alguna forma valida la teoría que fundamenta el instrumento.

2. Cuatro factores o constructos dan cuenta de la variabilidad del desempeño de la prueba.

3. Cada uno de los factores identificados y sus respectivos ítems presentan consistencia interna estimada por los índices de confiabilidad.

4. Se ha logrado elaborar los baremos de acuerdo a rangos percentiles según grado de estudio y sexo de la muestra estudiada. 


\section{REFERENCIAS BIBLIOGRÁFICAS}

Alonso Tapia, J., Montero García, I. y Mateos Sanz, M. (1992). Evaluación de los estilos atributivos: el cuestionario EMA-II. En Alonso Tapia: Motivar en la adolescencia: teoría, evaluación e intervención. Madrid: Universidad Autónoma de Madrid.

Alonso Tapia, J. y Arce Saez, E. (1992). Expectativas de control y motivación: el cuestionario ECO. En Alonso Tapia: Motivar en la adolescencia: teoría, evaluación e intervención. Madrid: Servicio de Publicaciones de la Universidad Autónoma de Madrid.

Alonso Tapia, J. y Sánchez Ferrer, J. (1992). Cuestionario MAPE-I: Motivación hacia aprendizaje. En Alonso Tapia: Motivar en la adolescencia: teoría, evaluación e intervención. Madrid: Servicio de publicaciones de la Universidad Autónoma de Madrid.

Alonso Tapia, J. y Sánchez García, F. (1992). Estilos atributivos y motivación: el cuestionario EAT. En Alonso Tapia: Motivar en la adolescencia: teoría, evaluación e intervención. Madrid: Servicio de publicaciones de la Universidad Autónoma de Madrid.

Bandura, A. (1987). Pensamiento y Acción. Fundamentos sociales. Barcelona: Martínez Roca.

Frydenberg, E. y Lewis, R. (1997). ACS: Escala de Afrontamiento para Adolescentes. Madrid: TEA.

García, F. y Musitu, G. (1999). AF5: Autoconcepto Forma 5. Madrid: TEA.

Gismero González, E. (2000). EHS: Escala de Habilidades Sociales. Madrid: TEA.

Kline, P. (1993). The Handbook of Psychological Testing. London: Routhledge.

Martín del Buey, F. (1997). Programa Integrado de Acción Tutorial. Revista de Orientación Educacional. Santiago de Chile: Universidad de Playa Ancha.

Martín del Buey, Zapico, Moris, Marcone y Dapello (2004). Evaluación de la personalidad eficaz en contextos educativos: primeros resultados. Revista orientación educacional. No 33-34, pp. 79-101.

Martín del Buey, F. (2000). Programa Integrado de Acción Tutorial: Marco Conceptual. Desarrollo de la Personalidad Eficaz en Contextos Educativos. Oviedo: Ediciones fmb2001.

Martín del Buey, F. Y Fernández Zapico, A. (2003). Programa de Desarrollo de la Personalidad Eficaz en Contextos Educativos: Primeros Resultados. Magíster, 19, 277-291. Oviedo: Universidad de Oviedo.

Nunnally y Jum, C. (1995). Teoría psicométrica. México: McGraw Gil. 3ra Edición 